\title{
Three-Fraction Intra-Cavitary Accelerated Partial Breast Brachytherapy: Early Provider and Patient-Reported Outcomes of a Novel Regimen
}

\author{
Krishan R. Jethwa, MD., Sean S. Park, MD., PhD., Karthik Gonuguntla, MBBS., Stephanie M. \\ Wick, DO., Laura A. Vallow, MD., Christopher L. Deufel, PhD., Thomas J. Whitaker, PhD., \\ Keith M. Furutani, PhD., Kathryn J. Ruddy, MD., Kimberly S. Corbin, MD., Tina J. Hieken, \\ MD., and Robert W. Mutter, MD. \\ Department of Radiation Oncology (Drs. Jethwa, Park, Gonuguntla, Wick, Vallow, Deufel, \\ Whitaker, Furutani, Corbin, Mutter), Division of Medical Oncology (Dr. Ruddy), Department of \\ Surgery (Dr. Hieken), Mayo Clinic, Rochester, Minnesota. Department of Radiation Oncology, \\ Mayo Clinic, Jacksonville, Florida (Dr. Vallow)
}

\begin{abstract}
Purpose/objectives: To report early adverse events and patient-reported outcomes (PROs) of 3fraction intracavitary catheter-based partial breast brachytherapy (ICBB).

Materials/methods: Eligible women $\geq 50$ years of age with $\mathcal{2} .5 \mathrm{~cm}$, lymph node negative invasive or in-situ breast cancer underwent breast conserving surgery and placement of a brachytherapy applicator. ICBB was initiated on the second weekday after surgery and prescribed to $21 \mathrm{~Gy}$ in three, once daily, fractions. Common Terminology Criteria for Adverse Events (CTCAE) v 4.0, 10-point Linear Analog Scales Assessment, PROs version of the CTCAE (PROCTCAE), and Harvard Breast Cosmesis Scale were utilized for provider and patient-reported assessments.
\end{abstract}

Results: 73 women were treated for invasive (79\%) or in-situ (21\%) breast cancer. The median time to completion of surgery and radiotherapy was 6 days. After 14 months median follow-up, two patients (3\%) had developed breast infections that resolved with oral antibiotics. There was no other treatment-associated adverse event $\geq$ grade 2 . The grade 1 seroma rate at 3 months was $20 \%$ which dropped to $8 \%$ at 12 months; none required intervention. At 12 months, $91 \%$ of patients reported an overall QoL score as $\geq 8$ out of 10 and patient-reported cosmesis was good or excellent in $95 \%$. All patients are alive without relapse at the last follow-up.

Conclusions: 3-fraction ICBB is associated with low rates of early provider and patient reported adverse events and compares favorably with early outcomes of more protracted ICBB regimens

Correspondence to: Robert W. Mutter, MD, Department of Radiation Oncology, Mayo Clinic, 200 First St SW, Rochester, MN 55905 (mutter.robert@ mayo.edu Phone: 507-284-8227 Fax: 507-284-0079).

Publisher's Disclaimer: This is a PDF file of an unedited manuscript that has been accepted for publication. As a service to our customers we are providing this early version of the manuscript. The manuscript will undergo copyediting, typesetting, and review of the resulting proof before it is published in its final citable form. Please note that during the production process errors may be discovered which could affect the content, and all legal disclaimers that apply to the journal pertain.

Conflicts of Interests:

None. 
including twice daily $(3.4 \mathrm{~Gy} \times 10)$ fractionation studied in NSABP B-39. Further investigation is warranted.

\section{Summary:}

We report outcomes of a prospective trial evaluating adjuvant 3-fraction intra-cavitary accelerated partial breast brachytherapy for women with early stage breast cancer. With early follow-up, we have identified low rates of adverse events, favorable patient-reported quality of life, and a high rate of excellent or good breast cosmesis. These data are promising and warrant further study.

\section{Keywords}

partial breast; breast cancer; radiotherapy; brachytherapy; APBI

\section{Introduction:}

Accelerated partial breast irradiation (APBI) is an established adjuvant treatment strategy for appropriately selected women electing breast conserving surgery (BCS) for early stage invasive breast cancer or ductal carcinoma in-situ (DCIS) [1]. This practice is supported by data from randomized controlled trials demonstrating non-inferior oncologic outcomes relative to whole breast irradiation (WBI) when APBI was delivered with varying techniques, doses, and fractionation regimens [2-4].

There is no current consensus on the ideal APBI treatment approach [3-11]. Intracavitary catheter-based partial breast brachytherapy (ICBB) is one attractive modality for APBI administration. ICBB enables a higher level of treatment conformality compared with external beam APBI techniques. At the same time, ICBB is less technically demanding than interstitial brachytherapy, which has resulted in broader dissemination of ICBB into breast cancer radiotherapy practice [12].

Compared with whole breast irradiation and external beam APBI, ICBB has been associated with a unique side effect profile, typified by higher rates of infection and seroma. In one large single institution experience the infection rate following ICBB was $14 \%$, with many infections occurring several weeks after the completion of therapy [13]. In addition, in retrospective population-based analyses, ICBB has been associated with an increased frequency of infectious and non-infectious complications compared with WBI [14, 15]. In North America, ICBB is most commonly administered in 10, twice daily fractions of $3.4 \mathrm{~Gy}$, which is also the regimen being investigated in the National Surgical Adjuvant Breast and Bowel Project (NSABP) B-39/Radiation Therapy Oncology Group (RTOG) 0413 trial. The relationship between the duration that ICBB treatment applicators are in place for radiotherapy delivery, and the rate of infectious and noninfectious complications of ICBB has yet to be investigated.

Recently, a meta-analysis of the UK Standardization of Breast Radiotherapy (START) A and START pilot trial demonstrated that breast cancer is relatively sensitive to large fraction sizes [16]. In that study, the $\alpha / \beta$ ratio for breast cancer locoregional relapse was estimated at 3.5. This value was numerically less than or equal to the $\alpha / \beta$ ratios for common late adverse 
events of breast radiotherapy including breast shrinkage, breast induration, telangiectasia, and breast edema. These data raised the possibility that further shortening of breast radiotherapy fractionation schedules could actually improve the therapeutic ratio of breast cancer radiotherapy.

Based upon these data, we hypothesized that a daily, 3-fraction ICBB treatment regimen would offer comparable local control as more commonly utilized APBI or WBI regimens, but may potentially offer a reduction in adverse events (AEs). We further hypothesized that shortening the time that the ICBB treatment applicator is in place following surgical resection may reduce the risk of infection and seroma. We elected daily fractionation based upon data suggesting that greater than 6 hours between fractions may be needed for completion of sublethal damage repair when larger fraction sizes are administered [17, 18].The purpose of this study is to report early physician-assessed AEs and patient-reported health-related quality of life (QoL) from our experience with a novel 3-fraction ICBB regimen.

\section{Methods and materials:}

\section{Study Population}

The study population included consecutive patients treated with 3-fraction ICBB for early stage breast cancer at the XXXX Clinic. MC1532 is a prospective, institutional review board approved study evaluating daily 3-fraction APBI utilizing intracavitary catheter-based brachytherapy, external beam 3-D conformal radiotherapy, or external beam pencil-beam scanning intensity modulated proton therapy. Treatment modality was chosen according to physician and patient discretion. Eligibility criteria included women age 50 years or older with pathologic tumor size $\leq 2.5 \mathrm{~cm}$, lymph node (LN) negative, estrogen receptor (ER)positive invasive ductal carcinoma or pure DCIS. Between 2015 and 2017, 56 patients were treated with ICBB on MC1532 (54 at XXXX, and 2 at XXXX). After enrollment to MC1532 was completed, an additional 17 patients were treated with 3-fraction ICBB on a prospective registry. These patients met the eligibility criteria of MC1532 and were all treated and followed according to the MC1532 protocol. Therefore, there were 73 evaluable patients included in this analysis of early physician-assessed AEs and patient-reported health-related QoL of patients treated with 3-fraction ICBB. Of note, the primary endpoint of MC1532 is the difference in the percentage of patients with adverse cosmesis (fair or poor cosmesis) at 3 years compared to baseline, as assessed by trained nurse providers and will be reported for all cohorts at a later date with further maturation of follow-up.

\section{Treatment Procedures}

Patients underwent BCS with placement of an intracavitary brachytherapy applicator at the initial operation if surgical margins and sentinel lymph nodes were negative on frozen section pathologic assessment, per our previously reported institutional practice [19]. A SAVI® (Cianna Medical, Aliso Viejo, CA) multi-channel intra-cavitary applicator was used in 71 patients, and a MammoSite $®$ (Hologic, Marlborough, MA) single-lumen intra-cavitary balloon applicator was used in 2 patients. A computed tomography (CT) simulation was performed the day following surgical resection, with treatment initiated post-operative day 2 
after final pathologic confirmation of treatment eligibility. Patients received oral antibiotic prophylaxis while the catheter was in place and antibiotics were discontinued following the final radiation fraction.

The planning target volume (PTV) included the lumpectomy cavity plus a $1 \mathrm{~cm}$ expansion, without extension into the chest wall, pectoralis muscle, or within $5 \mathrm{~mm}$ of the skin surface. The prescribed radiotherapy dose was $21 \mathrm{~Gy}$ in 3 , once daily, fractions utilizing a high-dose rate (HDR) brachytherapy afterloader (figure 1). Treatment was delivered on 3 consecutive business days, with applicator removal immediately following the final fraction.

Just prior to the initiation of our protocol, a whole breast irradiation regimen of $40 \mathrm{~Gy}$ in 15 fractions was demonstrated in the START B clinical trial to provide equivalent locoregional control as conventionally fractionated whole breast irradiation (i.e. $50 \mathrm{~Gy}$ in 25 fractions) but less toxicity [20]. Therefore, we sought to develop a 3 fraction ICBB regimen that would have a similar biological effect for tumor control as this reference schedule. Given the clinically favorable population anticipated in our trial and the low risk of local recurrence reported with hypofractionated whole breast irradiation alone in similar patients [20, 21], we elected to identify a regimen with a biologically equivalent effect of $40 \mathrm{~Gy}$ in 15 fractions alone without a lumpectomy cavity boost, an approach which has since been validated with publication of the IMPORT Low clinical trial [2]. We acknowledge that the linear quadratic model has been questioned for large doses per fraction and that alternative models have been proposed [22, 23]. However, our main focus was a simple linear quadratic model because it has decades of use in the radiotherapy field, is mathematically simple, and biological parameters other than $\alpha / \beta$ are not well established. The $\alpha / \beta$ estimate for tumor control in 10-year follow-up outcomes of the UK START pilot and START A trials was 3.5, the most robust data available to date on the fraction size sensitivity of breast cancer [20]. Forty Gy in 15 fractions is estimated to be equivalent to $44.7 \mathrm{~Gy}_{3.5}$ in 2 Gy fractions (EQD2). Taking into consideration the reduced time for repopulation [24] as well as the potential increased cell damage due to the higher dose gradients with brachytherapy [25], we elected a regimen of $7 \mathrm{~Gy} \times 3$ fractions, which translates to $40.1 \mathrm{~Gy}_{3.5} \mathrm{EQD} 2$. Of note, this regimen is the most commonly employed schedule for vaginal cuff brachytherapy and is associated with excellent local control and low rates of adverse events at that disease site [26]. Furthermore, the $\mathrm{BED}_{3.5}$ of this regimen is comparable to the BED of the investigational five fraction arms of the FAST Forward clinical trial as well as the brachytherapy regimen of $3.4 \mathrm{~Gy}$ adopted in NSABP B39/RTOG0413. However, we hypothesized we may observe less late toxicity with our daily fractionation regimen due to more complete inter-fraction recovery with daily, rather than twice daily fractionation [27, 28].

Planning objectives included a PTV D90\% (minimum dose covering 90\% of PTV) $>90 \%$ of prescription, PTV V150\% (volume of PTV receiving 150\% of the prescribed dose or more) $\leq 35 \mathrm{cc}$, and PTV V200\% (volume of PTV receiving 200\% of the prescribed dose or more) $\leq 10 \mathrm{cc}$.

Normal tissue planning objectives included, $5 \mathrm{~mm}$ from skin surface: maximum dose < $125 \%$ of prescription, skin surface: maximum dose $<100 \%$ of prescription, and chest wall (including ribs): maximum dose $<100 \%$ of prescription. 


\section{Adverse Events and Patient-Reported Outcomes Assessment}

Patients were prospectively assessed per the Common Terminology Criteria for Adverse Events (CTCAE) v 4.0 at baseline, at completion of brachytherapy, and in follow-up at 3 months, 12 months, and then annually. Patients also completed a breast cancer questionnaire which included a compilation of linear analog scale assessment (LASA) 10-point scales, Harvard Breast Cosmesis Scale (HBCS), and breast cancer therapy-related items from the patient-reported outcomes version of the Common Terminology Criteria for Adverse Events (PRO-CTCAE) reported here [30-33]. The LASA was utilized to assess patient-reported QoL (1 indicating "as bad as it can be" and 10 indicating "as good as it can be") and individual outcome measures of pain and fatigue ( 0 indicating "not at all" and 10 indicating "as bad as it can be"). The HBCS was used to collect patient-reports of breast cosmesis from a range of excellent, good, fair, or poor. The PRO-CTCAE was used to assess skin toxicity over the previous 7 days, using a 5- point scale ( 0 indicating "none" and 5 indicating "very severe").

All physician-assessed AEs and PROs were reported descriptively as the proportion of patients experiencing an event at baseline and at each follow-up time point.

\section{Results:}

Between June 2015 and October 2017, 73 patients met study inclusion and received 3fraction ICBB. The median follow-up was 14 months (interquartile range [IQR] 6, 22). Patient characteristics are shown in table 1. The median patient age was 66 (IQR 60, 70). The majority of patients had grade 1-2 (88\%), ER-positive (96\%), invasive carcinoma (79\%) of ductal histology (95\%) measuring $\leq 2 \mathrm{~cm}$ (95\%) with clinically (if DCIS) or pathologically (if invasive disease) negative lymph nodes (100\%). Sixty-four percent of patients received adjuvant endocrine therapy and none received chemotherapy.

The median PTV volume was $44 \mathrm{cc}$ (IQR 38.9, 49.2). Target and normal tissue dosimetric constraints and achieved parameters are displayed in table 2 . The median achieved dosimetric statistics included a PTV D90\% of 96.5\% (IQR 92.2, 99.9), skin max dose of $37.5 \%$ (IQR 24.3, 59.2), chest wall max dose of $89.4 \%$ (IQR 65.1, 102.6), and heart mean of 0.5 Gy (IQR 0.3, 0.8).

The median time to completion of all locoregional therapy (surgery and radiotherapy) was 6 days (IQR 4, 6).

Physician-assessed AEs, reported as the highest grade toxicity at each time-point, are displayed in table 3 . The grade 1 seroma rate at 3 months was $20 \%$ (11 of 56 evaluable patients), which dropped to $8 \%$ ( 4 of 49 ) at 12 months; none required intervention. By last follow-up, 2 (3\%) patients had developed a breast infection which resolved with the use of oral antibiotics. There was no other treatment-associated AE $\geq$ grade 2. No patients experienced an ipsilateral rib fracture.

Patient-reported outcomes are presented in table 4. At baseline, end of treatment, 3 months, and 12 months follow-up 77\%, 70\%, 79\%, and 71\% of evaluable patients, respectively, 
completed the patient reported outcomes survey. At 12 months, 91\% reported overall QoL as at least 8 out of 10, with 10 indicating quality of life "as good as it can be" and 0 indicating quality of life "as bad as it can be". Treatment also did not detrimentally impact patientreported fatigue or patient-reported pain. Patient-reported cosmesis was good or excellent in $95 \%$.

All patients are alive without relapse at the last follow-up.

\section{Discussion:}

The investigation of abbreviated breast radiotherapy regimens is attractive given recent evidence highlighting the fraction size sensitivity of breast cancer as well as increased emphasis on delivering value-based health care [16, 34]. Here-in, we report adverse events and patient-reported outcomes from a prospective evaluation of a novel 3-fraction ICBB regimen. With early follow-up, we have identified low rates of adverse events, favorable patient-reported quality of life, and a high rate of excellent or good breast cosmesis. These data are promising and warrant further study.

ICBB is a particularly appealing strategy for APBI delivery as ICBB provides high conformality to the lumpectomy cavity, limited normal tissue exposure, and relative ease of administration compared to other brachytherapy techniques. While the majority of data would support the efficacy of ICBB $[5,7,8,11,35]$, distinct complication risks have been associated with this technique. For example, breast infection rates of $8-16 \%$ have been reported with ICBB $[7,8,13,15]$, higher than what is typically seen with external beam radiotherapy. In recent retrospective reports of patients treated at our institution with intraoperative catheter placement and ICBB utilizing the widely adopted 10 fraction twice daily regimen, the breast infection rate was $6-11 \%[19,35]$. In a study from MD Anderson Cancer Center treated with that regimen, 13 of 91 patients (14\%) developed breast infection despite the routine use of prophylactic antibiotics, including five patients who underwent percutaneous aspiration, one patient who underwent incision and drainage, and four patients who required hospital admission [13]. In comparison with these prior experiences, just two patients developed infectious complications in our study that resolved with oral antibiotics. A potential source of infection is that ICBB requires an indwelling brachytherapy catheter for an extended duration. In the present study, the median total treatment time from surgery with intraoperative catheter placement until brachytherapy completion was reduced to 6 days, and only two infections were reported. Although further investigation is necessary, we postulate that the low rate of infectious complications in our study may in part be reflective of the reduced total time of an indwelling brachytherapy catheter made possible by the abbreviated treatment regimen.

An additional concern with ICBB has been the increased risk of symptomatic seroma. Vicini et al. reported outcomes of the MammoSite ${ }^{\circledR}$ breast brachytherapy registry, which represents one of the largest series of patients treated with ICBB reported to date, and identified a 13\% rate of symptomatic breast seroma [8]. The etiology of symptomatic seromas are likely multifactorial, but could be related to radiation dose, fractionation, short inter-fraction interval, lumpectomy cavity size, duration of catheter insertion, or other medical 
comorbidities. Promisingly, no patients have experienced symptomatic breast seroma requiring procedural intervention in our series to date.

In the Randomized Trial of Accelerated Partial breast Irradiation (RAPID) trial, which compared external beam APBI to WBI, a higher rate of fair or poor breast cosmesis, telangiectasia, and breast induration was identified following APBI delivered to $38.5 \mathrm{~Gy}$ in 10 fractions twice daily [29]. This is the same dose fractionation regimen being investigated for patients treated with external beam APBI in the NSABP B39 study, results of which are awaited. One explanation for the observed deterioration in cosmesis, acknowledge by the RAPID trial investigators and others, was that the volume of breast tissue irradiated with three-dimensional conformal external beam radiation therapy (3D-CRT) may have been too large $[29,36]$. However, in the more recently published UK IMPORT LOW trial, which maintained equivalent dose fractionation regimens amongst the comparative whole breast and generous volume partial breast 3D-CRT treatment groups, fewer late adverse effects were observed with APBI [2].

Another possible explanation for the deterioration in cosmesis observed with APBI in the RAPID trial is that the prescription dose resulted in a higher biologically effective dose for late effects than the WBI control arm. One potential contributor to the greater biologic effect is that the 6-hour interval between fractions may be insufficient to allow for complete sublethal damage repair $[17,18]$. Indeed, in the Continuous Hyperfractionated Accelerated Radiotherapy Trial (CHART) for head and neck squamous cell carcinoma, repair half times for skin telangiectasia and subcutaneous fibrosis, clinically relevant endpoints in breast cancer radiotherapy, were estimated to be 3.8 hours and 4.4 hours, respectively [37]. These data would suggest that a 6 -hour interval between fractions would only allow for $\sim 65 \%$ repair of sublethal damage, with potentially important clinical implications when relatively large fraction sizes are administered as with APBI [17, 18]. Although late AEs of radiotherapy can occur decades out from treatment, clinically significant differences in adverse cosmesis and late toxicity were observed by 3 years in the RAPID trial [38]. Of note, the magnitude of difference in late toxicity between treatment arms in that study at 5 years and 3 years were similar. Therefore, for early phase clinical trials testing novel fractionation regimens for breast cancer where late toxicity is of primary interest, follow-up of 3 years may provide adequate evidence to support or refute whether further investigation may be warranted in larger phase 3 randomized studies.

Relatedly, Wilkinson et al. previously reported the results of a prospective phase I/II trial of intra-cavitary balloon-based ICBB to a dose of 28 Gy in 4, twice daily fractions, over 2 days [39]. At 3 years there was a $7 \%$ rate of ipsilateral rib fracture, $7 \%$ incidence of symptomatic seroma requiring aspiration, 7\% incidence of grade 2 fibrosis, and $4 \%$ incidence of grade 2 talengiectasia with that regimen. Although we speculate that less late toxicity will be observed with our regimen, given the lower biologically effective dose of $21 \mathrm{~Gy}$ in 3 fractions, further follow-up will be needed to better delineate late adverse events of this approach. In addition to the dose-fractionation schedule, it is possible that improved skin and chest wall dosimetry provided by multi-channel brachytherapy applicator, which was used in all but two patients in our study, will enable improved outcomes with ultra hypofractionated ICBB [40, 41]. Indeed, in our series, we achieved a maximum dose to the 
most superficial $5 \mathrm{~mm}$ of the skin of 53\%. The favorable skin dosimetry may be partially attributable to the use of multi-channel ICBB catheters and high prioritization on skin sparing during treatment planning. However, physicians may also have preferentially opted for available external beam APBI techniques for superficially located tumors. Optimal patient selection for the various APBI treatment modalities available is an important area for future investigation.

Post-operative stereotactic body radiation therapy (SBRT) and other ultra-hypofractionated external beam radiotherapy techniques are also under investigation [4, 42, 43]. Livi et al. have reported on 520 women with early stage breast cancer who received either whole breast irradiation to a dose of $50 \mathrm{~Gy}$ in 25 daily fractions, or APBI to a dose of $30 \mathrm{~Gy}$, delivered in 5 non-consecutive daily fractions, targeting the lumpectomy cavity with an approximate 2 $\mathrm{cm}$ expansion to PTV, delivered with external beam intensity modulated radiotherapy. At 5years, they identified non-inferior local control, a reduction in acute adverse events, and a $100 \%$ rate of excellent or good breast cosmesis in the APBI treatment group. These serve as promising data, supporting further investigation of ultrahypofractionated external beam techniques in the treatment of women with early stage breast cancer.

Although promising, there are limitations to this work. This was a single arm study and all patients underwent surgical resection and ICBB at two XXXX treatment sites. Early AEs and QoL outcomes appear promising, however, further follow-up is required to ensure acceptable local control, and to allow for continued monitoring of evolving changes in late AEs and breast cosmesis [29]. In our study, patients completed both breast surgery and adjuvant radiotherapy in as little as 4 days. Whether such a condensed treatment schedule can increase access to radiotherapy and improve breast conservation rates and other treatment outcomes in resource constrained settings is a potentially important area for future investigation [44].

\section{Conclusion:}

Treatment of patients with early stage breast cancer using a novel 3-fraction, once daily, ICBB treatment regimen is associated with favorable physician-assessed AEs, PROs, and breast cosmesis at early follow-up. These results compare favorably with early outcomes of more protracted ICBB regimens, including the twice daily $(3.4 \mathrm{~Gy} \times 10)$ fractionation studied in NSABP B-39. A randomized trial comparing these regimens is warranted.

\section{Acknowledgments}

Funding:

This research was supported by the Lawrence W. and Marilyn Matteson Fund in Cancer Research and K12 HD065987 (Robert W. Mutter)

\section{Abbreviations}
APBI
accelerated partial breast irradiation
BCS
breast conserving surgery 


\begin{tabular}{|c|c|}
\hline DCIS & ductal carcinoma in-situ \\
\hline WBI & whole breast irradiation \\
\hline ICBB & intracavitary catheter-based partial breast brachytherapy \\
\hline NSABP & National Surgical Adjuvant Breast and Bowel Project \\
\hline RTOG & Radiation Therapy Oncology Group \\
\hline UK START & United Kingdom Standardization of Breast Radiotherapy \\
\hline AEs & adverse events \\
\hline PROs & patient-reported outcomes \\
\hline QoL & quality of life \\
\hline $\mathbf{L N}$ & lymph node \\
\hline ER & estrogen receptor \\
\hline CT & computed tomography \\
\hline PTV & planning target volume \\
\hline HDR & high-dose rate \\
\hline DX\% & minimum dose covering $\mathrm{X} \%$ of the target \\
\hline VX\% & $\begin{array}{l}\text { percentage volume of target or organ receiving } \mathrm{X} \% \text { of the prescribed } \\
\text { dose or more }\end{array}$ \\
\hline CTCAE & Common Terminology Criteria for Adverse Events \\
\hline LASA & linear analog scale assessment \\
\hline HBCS & Harvard Breast Cosmesis Scale \\
\hline PRO-CTCAE & $\begin{array}{l}\text { patient-reported outcomes version of the Common Terminology } \\
\text { Criteria for Adverse Events }\end{array}$ \\
\hline IQR & interquartile range \\
\hline RAPID & Canadian Randomized Trial of Accelerated Partial Breast Irradiation \\
\hline 3D-CRT & three-dimensional conformal external beam radiation therapy \\
\hline CHART & Continuous Hyperfractionated Accelerated Radiotherapy Trial \\
\hline SBRT & Stereotactic Body Radiation Therapy \\
\hline
\end{tabular}




\section{References}

1. Correa C, et al., Accelerated Partial Breast Irradiation: Executive summary for the update of an ASTRO Evidence-Based Consensus Statement. Pract Radiat Oncol, 2017 7(2): p. 73-79. [PubMed: 27866865]

2. Coles CE, et al., Partial-breast radiotherapy after breast conservation surgery for patients with early breast cancer (UK IMPORT LOW trial): 5-year results from a multicentre, randomised, controlled, phase 3, non-inferiority trial. Lancet, 2017 390(10099): p. 1048-1060. [PubMed: 28779963]

3. Strnad V, et al., 5-year results of accelerated partial breast irradiation using sole interstitial multicatheter brachytherapy versus whole-breast irradiation with boost after breast-conserving surgery for low-risk invasive and in-situ carcinoma of the female breast: a randomised, phase 3 , non-inferiority trial. Lancet, 2016 387(10015): p. 229-38. [PubMed: 26494415]

4. Livi L, et al., Accelerated partial breast irradiation using intensity-modulated radiotherapy versus whole breast irradiation: 5-year survival analysis of a phase 3 randomised controlled trial. Eur J Cancer, 2015 51(4): p. 451-63. [PubMed: 25605582]

5. White J, et al., Long-Term Cancer Outcomes From Study NRG Oncology/RTOG 9517: A Phase 2 Study of Accelerated Partial Breast Irradiation With Multicatheter Brachytherapy After Lumpectomy for Early-Stage Breast Cancer. Int J Radiat Oncol Biol Phys, 2016 95(5): p. 14601465. [PubMed: 27479725]

6. Rabinovitch R, et al., Long-Term Update of NRG Oncology RTOG 0319: A Phase 1 and 2 Trial to Evaluate 3-Dimensional Conformal Radiation Therapy Confined to the Region of the Lumpectomy Cavity for Stage I and II Breast Carcinoma. Int J Radiat Oncol Biol Phys, 2016 96(5): p. 10541059. [PubMed: 27869081]

7. Cuttino LW, et al., Long-term results from the Contura multilumen balloon breast brachytherapy catheter phase 4 registry trial. Int J Radiat Oncol Biol Phys, 2014 90(5): p. 1025-9. [PubMed: 25442036]

8. Vicini F, et al., Five-year analysis of treatment efficacy and cosmesis by the American Society of Breast Surgeons MammoSite Breast Brachytherapy Registry Trial in patients treated with accelerated partial breast irradiation. Int J Radiat Oncol Biol Phys, 2011 79(3): p. 808-17. [PubMed: 20472364]

9. Veronesi U, et al., Intraoperative radiotherapy versus external radiotherapy for early breast cancer (ELIOT): a randomised controlled equivalence trial. Lancet Oncol, 2013 14(13): p. 1269-77. [PubMed: 24225155]

10. Vaidya JS, et al., Targeted intraoperative radiotherapy versus whole breast radiotherapy for breast cancer (TARGIT-A trial): an international, prospective, randomised, non-inferiority phase 3 trial. Lancet, 2010 376(9735): p. 91-102. [PubMed: 20570343]

11. Marta GN, et al., Accelerated partial irradiation for breast cancer: systematic review and metaanalysis of 8653 women in eight randomized trials. Radiother Oncol, 2015 114(1): p. 42-9. [PubMed: 25480094]

12. Cox JA and Swanson TA, Current modalities of accelerated partial breast irradiation. Nature Reviews Clinical Oncology, 2013 10: p. 344.

13. Haynes AB, et al., Timing of infectious complications following breast-conserving therapy with catheter-based accelerated partial breast irradiation. Ann Surg Oncol, 2014 21(8): p. 2512-6. [PubMed: 24736987]

14. Presley CJ, et al., Patterns of use and short-term complications of breast brachytherapy in the national medicare population from 2008-2009. J Clin Oncol, 2012 30(35): p. 4302-7. [PubMed: 23091103]

15. Smith GL, et al., Association between treatment with brachytherapy vs whole-breast irradiation and subsequent mastectomy, complications, and survival among older women with invasive breast cancer. Jama, 2012 307(17): p. 1827-37. [PubMed: 22550197]

16. Haviland JS, et al., The UK Standardisation of Breast Radiotherapy (START) trials of radiotherapy hypofractionation for treatment of early breast cancer: 10-year follow-up results of two randomised controlled trials. The lancet oncology, 2013 14(11): p. 1086-1094. [PubMed: 24055415] 
17. Bentzen SM, Saunders MI, and Dische S, Repair halftimes estimated from observations of treatment-related morbidity after CHART or conventional radiotherapy in head and neck cancer. Radiother Oncol, 1999 53(3): p. 219-26. [PubMed: 10660202]

18. Bentzen SM and Yarnold JR, Reports of unexpected late side effects of accelerated partial breast irradiation--radiobiological considerations. Int J Radiat Oncol Biol Phys, 2010 77(4): p. 969-73. [PubMed: 20610037]

19. Hieken TJ, et al., A Novel Treatment Schedule for Rapid Completion of Surgery and Radiation in Early-Stage Breast Cancer. Ann Surg Oncol, 2016 23(10): p. 3297-303. [PubMed: 27334215]

20. Haviland JS, et al., The UK Standardisation of Breast Radiotherapy (START) trials of radiotherapy hypofractionation for treatment of early breast cancer: 10-year follow-up results of two randomised controlled trials. The lancet oncology, 2013 14(11): p. 1086-94. [PubMed: 24055415]

21. Whelan TJ, et al., Long-term results of hypofractionated radiation therapy for breast cancer. The New England journal of medicine, 2010 362(6): p. 513-20. [PubMed: 20147717]

22. Allen Li X, et al., The use and QA of biologically related models for treatment planning: short report of the TG-166 of the therapy physics committee of the AAPM. Med Phys, 2012 39(3): p. 1386-409. [PubMed: 22380372]

23. Wang JZ, et al., A generalized linear-quadratic model for radiosurgery, stereotactic body radiation therapy, and high-dose rate brachytherapy. Sci Transl Med, 2010 2(39): p. 39 ra48.

24. Wyatt RM, Jones BJ, and Dale RG, Radiotherapy treatment delays and their influence on tumour control achieved by various fractionation schedules. Br J Radiol, 2008 81(967): p. 549-63. [PubMed: 18378526]

25. Dale RG, et al., Calculation of integrated biological response in brachytherapy. Int J Radiat Oncol Biol Phys, 1997 38(3): p. 633-42. [PubMed: 9231690]

26. Harkenrider MM, et al., American Brachytherapy Task Group Report: Adjuvant vaginal brachytherapy for early-stage endometrial cancer: A comprehensive review. Brachytherapy, 2017 16(1): p. 95-108. [PubMed: 27260082]

27. Bentzen SM, Saunders MI, and Dische S, Repair halftimes estimated from observations of treatment-related morbidity after CHART or conventional radiotherapy in head and neck cancer. Radiotherapy and oncology : journal of the European Society for Therapeutic Radiology and Oncology, 1999 53(3): p. 219-26. [PubMed: 10660202]

28. Bentzen SM and Yarnold JR, Reports of unexpected late side effects of accelerated partial breast irradiation--radiobiological considerations.International journal of radiation oncology, biology, physics, 2010 77(4): p. 969-73.

29. Olivotto IA, et al., Interim cosmetic and toxicity results from RAPID: a randomized trial of accelerated partial breast irradiation using three-dimensional conformal external beam radiation therapy. J Clin Oncol, 2013 31(32): p. 4038-45. [PubMed: 23835717]

30. Harris JR, et al., Analysis of cosmetic results following primary radiation therapy for stages I and II carcinoma of the breast. Int J Radiat Oncol Biol Phys, 1979 5(2): p. 257-61. [PubMed: 110740]

31. Locke DE, et al., Validation of single-item linear analog scale assessment of quality of life in neuro-oncology patients. J Pain Symptom Manage, 2007 34(6): p. 628-38. [PubMed: 17703910]

32. Basch E, et al., Development of the National Cancer Institute's patient-reported outcomes version of the common terminology criteria for adverse events (PRO-CTCAE). J Natl Cancer Inst, 2014 106(9).

33. Dueck AC, et al., Validity and Reliability of the US National Cancer Institute's Patient-Reported Outcomes Version of the Common Terminology Criteria for Adverse Events (PRO-CTCAE). JAMA Oncol, 2015 1(8): p. 1051-9. [PubMed: 26270597]

34. Young RC, Value-Based Cancer Care. N Engl J Med, 2015 373(27): p. 2593-5. [PubMed: 26580243]

35. Jethwa KR, et al., Patient-reported outcomes of catheter-based accelerated partial breast brachytherapy and whole breast irradiation, a single institution experience. Breast Cancer Res Treat, 2018.

36. Jagsi R, et al., Unacceptable cosmesis in a protocol investigating intensity-modulated radiotherapy with active breathing control for accelerated partial-breast irradiation. Int J Radiat Oncol Biol Phys, 2010 76(1): p. 71-8. [PubMed: 19409733] 
37. Dische S, et al., A randomised multicentre trial of CHART versus conventional radiotherapy in head and neck cancer. Radiother Oncol, 1997 44(2): p. 123-36. [PubMed: 9288840]

38. Olivotto IA, et al., Interim Cosmetic and Toxicity Results From RAPID: A Randomized Trial of Accelerated Partial Breast Irradiation Using Three-Dimensional Conformal External Beam Radiation Therapy. Journal of clinical oncology : official journal of the American Society of Clinical Oncology, 2013.

39. Wilkinson JB, et al., Four-year results using balloon-based brachytherapy to deliver accelerated partial breast irradiation with a 2-day dose fractionation schedule. Brachytherapy, 2012 11(2): p. 97-104. [PubMed: 21727032]

40. Bitter SM, et al., WBRT vs. APBI: an interim report of patient satisfaction and outcomes. J Contemp Brachytherapy, 2016 8(1): p. 17-22. [PubMed: 26985193]

41. Manoharan SR, et al., Dosimetry evaluation of SAVI-based HDR brachytherapy for partial breast irradiation. J Med Phys, 2010 35(3): p. 131-6. [PubMed: 20927219]

42. Meattini I, et al., Accelerated partial breast irradiation using intensity modulated radiotherapy versus whole breast irradiation: Health-related quality of life final analysis from the Florence phase 3 trial. Eur J Cancer, 2017 76: p. 17-26. [PubMed: 28262584]

43. Rahimi A, et al., Preliminary Results of a Phase 1 Dose-Escalation Trial for Early-Stage Breast Cancer Using 5-Fraction Stereotactic Body Radiation Therapy for Partial-Breast Irradiation. Int J Radiat Oncol Biol Phys, 2017 98(1): p. 196-205.e2. [PubMed: 28586960]

44. Khan AJ, et al., Nation-Scale Adoption of Shorter Breast Radiation Therapy Schedules Can Increase Survival in Resource Constrained Economies: Results From a Markov Chain Analysis. Int J Radiat Oncol Biol Phys, 2017 97(2): p. 287-295. [PubMed: 27986343] 

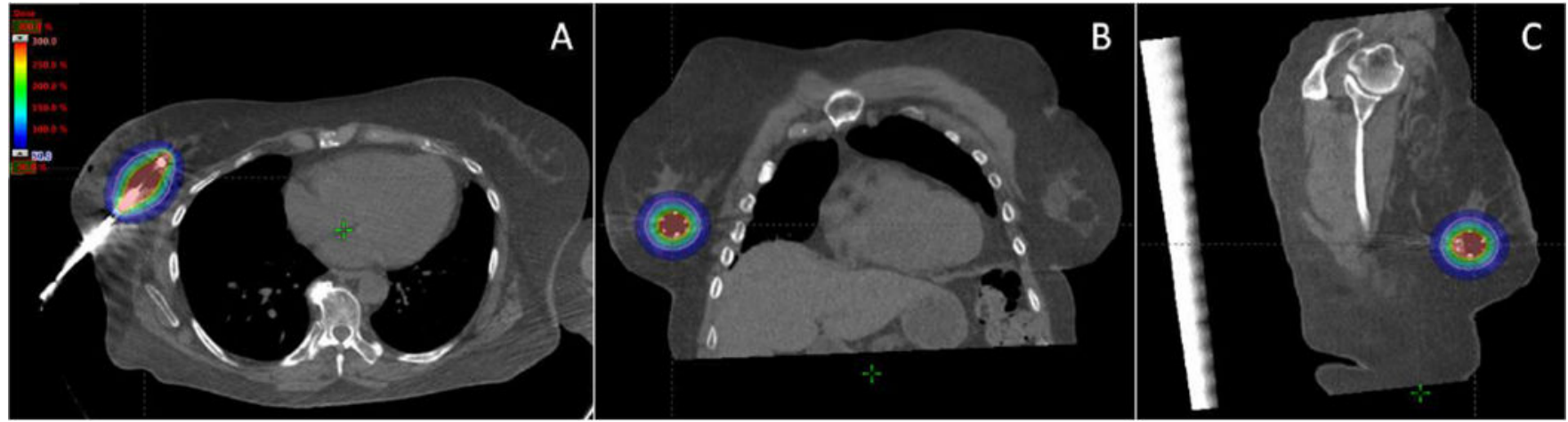

Figure 1:

Axial (A), coronal (B), and sagittal (C) images with dose color-wash (1050 cGy, 50\% of prescription) demonstrating APBI technique with intra-operatively placed multi-channel brachytherapy applicator. The planning target volume (pink) is shown. 
Table 1:

\section{Patient Characteristics}

\begin{tabular}{|c|c|c|}
\hline Variable $(n=73)$ & & Value* \\
\hline Age, median (IQR) & & $66(60-70)$ \\
\hline Follow-up & & $14(6-22)$ \\
\hline \multirow{2}{*}{ Laterality } & Right & $40(55 \%)$ \\
\hline & Left & $33(45 \%)$ \\
\hline \multirow{3}{*}{ Histology } & Ductal & $69(95 \%)$ \\
\hline & Lobular & $1(1 \%)$ \\
\hline & Other & $3(4 \%)$ \\
\hline Invasive & & $58(79 \%)$ \\
\hline DCIS & & $15(21 \%)$ \\
\hline \multirow{3}{*}{ Grade } & 1 & $29(40 \%)$ \\
\hline & 2 & $35(48 \%)$ \\
\hline & 3 & $9(12 \%)$ \\
\hline \multirow{3}{*}{ Tumor size } & $\leq 1 \mathrm{~cm}$ & $48(66 \%)$ \\
\hline & $>1-2 \mathrm{~cm}$ & $21(29 \%)$ \\
\hline & $>2-2.5 \mathrm{~cm}$ & $4(5 \%)$ \\
\hline LN negative & & $73(100 \%)$ \\
\hline \# LNs removed & & $2(1-3)$ \\
\hline \multirow{2}{*}{ ER status } & Positive & $70(96 \%)$ \\
\hline & Negative & $3(4)$ \\
\hline Her-2 Positive & & $1(2 \%)$ \\
\hline Adjuvant Endocrine therapy & & $47(64 \%)$ \\
\hline Adjuvant Chemotherapy & & $0(0 \%)$ \\
\hline
\end{tabular}

Continuous variables are reported as median (IQR). Categorical variables reported as number (\%). 
Table 2:

Dosimetric constraints and achieved parameters

\begin{tabular}{|c|c|c|c|}
\hline Target/Organ at Risk & DVH Parameter & Planning Constraint & Achieved Parameter Median (IQR) \\
\hline PTV volume & & & $44.0(38.9,49.2)$ \\
\hline \multirow{3}{*}{$P T V$} & D90\% (\%) & $\geq 90 \%$ & $96.5(92.2,99.9)$ \\
\cline { 2 - 4 } & V150\% (cc) & $\leq 35 \mathrm{cc}$ & $18.4(15.7,20.3)$ \\
\cline { 2 - 4 } & V200\% (cc) & $\leq 10 \mathrm{cc}$ & $9.4(8.4,10.3)$ \\
\hline Skin - 5 mm & Max (\%) & $<125 \%$ & $53.4(31.8,90.7)$ \\
\hline \multirow{2}{*}{ Skin } & Max (\%) & $<100 \%$ & $37.5(24.3,59.2)$ \\
\hline Chest wall & Max (\%) & $<100 \%$ & $89.4(65.1,102.6)$ \\
\hline \multirow{2}{*}{ Heart } & D0.01 cc (\%) & & $7.7(5.4,16.1)$ \\
\cline { 2 - 4 } & Mean (Gy) & & $0.5(0.3,0.8)$ \\
\hline
\end{tabular}

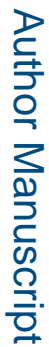


Table 3:

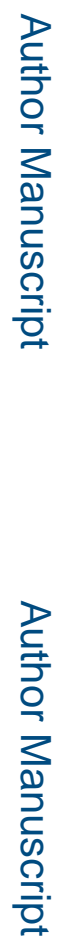

Physician-assessed adverse events

\begin{tabular}{|c|c|c|c|c|c|}
\hline Physician Assessed AEs & CTCAE Grade & Baseline (n=73) & Post-RT (n=73) & 3 month (n=56) & $\mathbf{1 2}$ month (n=49) \\
\hline Dermatitis & 1 & $3 \%$ & $21 \%$ & $5 \%$ & $0 \%$ \\
\hline Skin Hyperpigmentation & 1 & $3 \%$ & $7 \%$ & $16 \%$ & $14 \%$ \\
\hline Telangiectasia & 1 & $1 \%$ & $0 \%$ & $0 \%$ & $0 \%$ \\
\hline Superficial Fibrosis & 1 & $1 \%$ & $3 \%$ & $7 \%$ & $6 \%$ \\
\hline Deep fibrosis & 1 & $0 \%$ & $0 \%$ & $7 \%$ & $8 \%$ \\
\hline Seroma & 1 & $8 \%$ & $23 \%$ & $20 \%$ & $8 \%$ \\
\hline Breast pain & 1 & $5 \%$ & $1 \%$ & $4 \%$ & $0 \%$ \\
\hline Limb Edema & 1 & $0 \%$ & $0 \%$ & $0 \%$ & $0 \%$ \\
\hline Breast Edema & 1 & $1 \%$ & $11 \%$ & $18 \%$ & $0 \%$ \\
\hline Pneumonitis & 1 & $0 \%$ & $0 \%$ & $0 \%$ & $0 \%$ \\
\hline Brachial Plexopathy & 1 & $0 \%$ & $0 \%$ & $0 \%$ & $0 \%$ \\
\hline
\end{tabular}


Table 4:

Patient-Reported Outcomes

\begin{tabular}{|c|c|c|c|c|c|}
\hline PROs & Assessment $^{a \beta \epsilon}$ & Baseline $(n=56)$ & Post-RT $(\mathrm{n}=51)$ & 3 month $(n=44)$ & 12 month $(n=35)$ \\
\hline \multirow{3}{*}{ Quality of Life $^{a}$} & $8-10$ & $95 \%$ & $96 \%$ & $89 \%$ & $91 \%$ \\
\hline & $4-7$ & $4 \%$ & $2 \%$ & $7 \%$ & $9 \%$ \\
\hline & $1-3$ & $1 \%$ & $2 \%$ & $4 \%$ & $0 \%$ \\
\hline \multirow{4}{*}{ Breast Cosmesis $€$} & Excellent & $55 \%$ & $41 \%$ & $50 \%$ & $69 \%$ \\
\hline & Good & $45 \%$ & $49 \%$ & $39 \%$ & $26 \%$ \\
\hline & Fair & $0 \%$ & $10 \%$ & $9 \%$ & $6 \%$ \\
\hline & Poor & $0 \%$ & $0 \%$ & $2 \%$ & $0 \%$ \\
\hline \multirow{3}{*}{ Pain ${ }^{\beta}$} & $0-3$ & $61 \%$ & $69 \%$ & $86 \%$ & $86 \%$ \\
\hline & $4-6$ & $32 \%$ & $22 \%$ & $7 \%$ & $9 \%$ \\
\hline & $7-10$ & $7 \%$ & $10 \%$ & $7 \%$ & $6 \%$ \\
\hline \multirow{3}{*}{ Fatigue $^{\beta}$} & $0-3$ & $61 \%$ & $55 \%$ & $75 \%$ & $71 \%$ \\
\hline & $4-6$ & $25 \%$ & $37 \%$ & $16 \%$ & $12 \%$ \\
\hline & $7-10$ & $14 \%$ & $8 \%$ & $9 \%$ & $17 \%$ \\
\hline \multirow{5}{*}{ Skin Burns ${ }^{*}$} & None & $100 \%$ & $90 \%$ & $88 \%$ & $100 \%$ \\
\hline & Mild & $0 \%$ & $8 \%$ & $12 \%$ & $0 \%$ \\
\hline & Moderate & $0 \%$ & $0 \%$ & $0 \%$ & $0 \%$ \\
\hline & Severe & $0 \%$ & $0 \%$ & $0 \%$ & $0 \%$ \\
\hline & Very Severe & $0 \%$ & $2 \%$ & $0 \%$ & $0 \%$ \\
\hline
\end{tabular}

$a_{\text {LASA: }}$ Reported on a scale of 1-10 (1 indicating "as bad as it can be" and 10 indicating "as good as it can be")

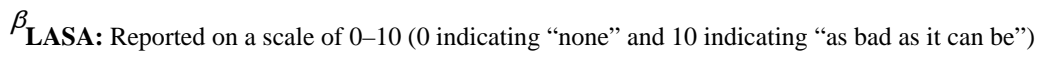

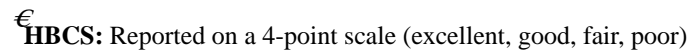

PRO-CTCAE: Reported on a 5-point scale (none, mild, moderate, severe, very severe) 